Original Paper

\title{
Effect of Pressure on Biodiesel Production in Supercritical Tert-butyl Methyl Ether (MTBE)
}

\author{
Obie FAROBIE ${ }^{* 1}$ and Yukihiko MATSUMURA $* 2$ \\ (Received November 5, 2014)
}

\begin{abstract}
A continuous reactor was used to examine the effect of pressure on biodiesel production using a supercritical tert-butyl methyl ether (MTBE) at the pressure range of $10-30 \mathrm{MPa}$ and temperature range of $300-400^{\circ} \mathrm{C}$. The effect of pressure on reaction rate as well as final product composition was negligible for the conditions employed here. This negligible effect could be explained by the almost constant density of MTBE. The pre-exponential factors and activation energy for each reaction step as well as its reverse reaction have been determined.
\end{abstract}

\section{Key Words}

Biomass, Biodiesel, MTBE, GTBE, Pressure effect

\section{Introduction}

To date, research on renewable energy has increased due to the worldwide energy problems of global warming, air pollution, and the depletion of fossil fuels. Biodiesel, which is derived mainly from biomass such as vegetable oil, animal fats, and microalgae, is a promising candidate for renewable energy. Biodiesel has low particulate matter and $\mathrm{CO}$ exhaust emission, it has a high flash point, and its physico-chemical properties are similar to those of conventional fuel ${ }^{12}{ }^{2}$. For these reasons, there is a high potential for biodiesel to be used as a substitute for petroleum diesel fuel.

In developing countries such as Indonesia and Thailand, biodiesel is produced mainly via a homogeneous alkali-catalyzed transesterification process due to the wide availability of catalysts such as potassium and sodium hydroxide. However, this method is problematic due to the sensitivity of these catalysts to the presence of water and free fatty acids (FFAs) of feedstock, which results in a saponification reaction and lower biodiesel yield ${ }^{3)}$. Several approaches have been used to overcome this problem,

※ 1 Department of Mechanical Science and Engineering, Hiroshima University

1-4-1, Kagamiyama, Higashi-Hiroshima-shi, Hiroshima 739-8527 Japan

※2 Division of Energy and Environmental Engineering, Institute of Engineering, Hiroshima University 1-4-1 Kagamiyama, Higashi-Hiroshima-shi, Hiroshima 739-8527 Japan including a two-step method in which acid and alkaline catalysts are applied in turn, lipase catalyst utilization, and solid catalyst utilization ${ }^{4}$.

Another approach involves the utilization of supercritical alcohol. Saka' s group at Kyoto University has published a series of pioneering studies ${ }^{5}$. Our laboratory has developed a method for non-catalytic biodiesel production using supercritical tert-butyl methyl ether $(\mathrm{MTBE})^{6}$. This novel method could not only overcome the problem of saponification and lower yield, but it could also produce a higher value by-product, i.e., glycerol tert-butyl ether (GTBE). GTBE has a higher value than glycerol because it has a high cetane number and a good ability to blend with diesel fuel ${ }^{7)}{ }^{8}$.

However, the effect of pressure on biodiesel yield using supercritical MTBE has not been sufficiently examined. Previous studies on biodiesel production with supercritical alcohol illustrated the specific effect of pressure. For supercritical methanol, He et al. ${ }^{9)}$ investigated the effect of pressure at $280^{\circ} \mathrm{C}$ and an oil-tomethanol molar ratio of 1:42. They reported that pressure significantly affected the yield in the pressure range from ambient pressure to $25 \mathrm{MPa}$. Above $25 \mathrm{MPa}$, the effect of pressure on biodiesel yield was negligible. Warabi et al. ${ }^{10)}$ also investigated the effect of pressure on supercritical alcohols. They found that at a temperature of $300{ }^{\circ} \mathrm{C}$, the effect of the reaction pressure did not result in a significant 
increase in reaction rate when the reaction pressure was higher than $20 \mathrm{MPa}$. For supercritical dimethyl carbonate, Ilham and Saka ${ }^{11)}$ found that the fatty acid methyl ester (FAME) yield increased with a pressure rise from 5 to 20 $\mathrm{MPa}$ at a constant temperature of $300^{\circ} \mathrm{C}$, but the increase was negligible at a pressure above $20 \mathrm{MPa}$. Goembira and Saka ${ }^{12)}$ also investigated the effect of pressure on FAME yield using supercritical methyl acetate. They found that the conversion of oil to biodiesel increased as the pressure rose from 5 to $20 \mathrm{MPa}$, but the conversion remained constant when pressure was increased from 20 to $30 \mathrm{MPa}$. Thus, it appears that at lower pressure, a pressure increase results in an increase in FAME yield, but at high pressure, a pressure increase no longer affects the yield. The investigation of the pressure effect is essential for biodiesel production under supercritical conditions, but as of yet there is no report on supercritical MTBE. Therefore, the purpose of this study is to obtain a better understanding of the effect of pressure on biodiesel yield using supercritical MTBE.

\section{Materials and Methods}

\subsection{Experimental apparatus and procedure}

The experimental apparatus consisted of a high pressure pump, a spiral reactor, a ceramic microheater, a filter, and a back-pressure regulator. This apparatus is schematically illustrated in Fig. 1.

The spiral reactor was made of stainless-steel tubing (SS316) with an inner diameter of $2.17 \mathrm{~mm}$. Thermocouples, connected with a tee union fitting, were equipped to measure temperatures inside the spiral reactor. The length of the reactor was $10.0 \mathrm{~m}$ and that of the heat exchanger was $2.5 \mathrm{~m}$. The heat exchanger allowed for rapid heating up and cooling down of the flow by exchanging heat between them.

The feedstocks, which consisted of canola oil and MTBE, were fed to the spiral reactor at the desired temperature. In this study, the transesterification process was carried out at various pressures of $10-30 \mathrm{MPa}$ in the temperature range $300-400^{\circ} \mathrm{C}$ with a fixed oil-to-MTBE molar ratio of 1:40. The reaction was carried out over 3-30 min, and samples were obtained after achieving a steady state. Note that the critical temperature and pressure of MTBE are $223.85^{\circ} \mathrm{C}$ and $3.448 \mathrm{MPa}$, respectively. After passing through the filter and back-pressure regulator,

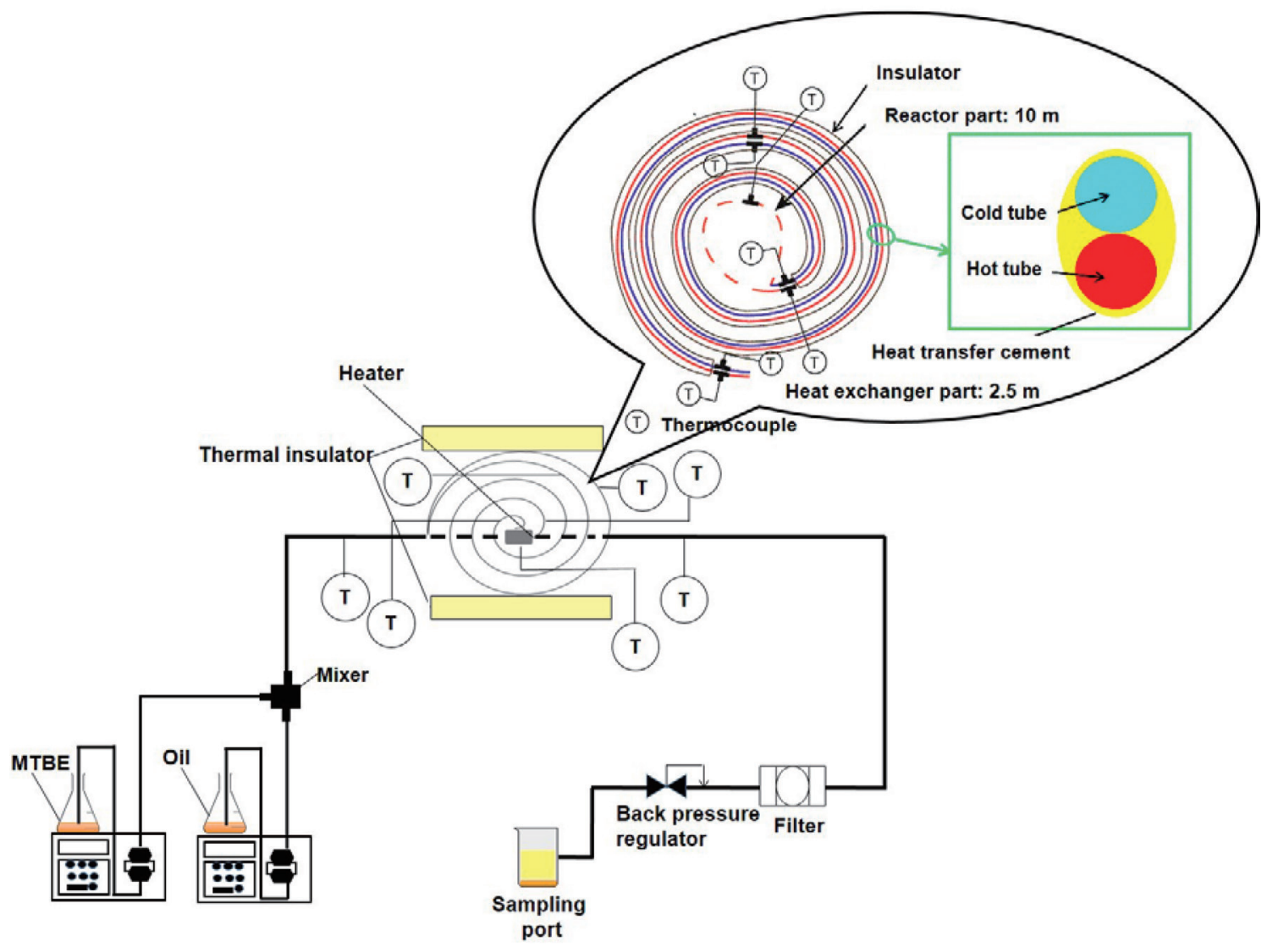

Fig. 1 Experimental apparatus 
Table 1 Experimental conditions for effect of pressure on biodiesel production in supercritical MTBE using spiral reactor

\begin{tabular}{ll}
\hline Types of condition & Experimental range \\
\hline Feedstock & Canola oil and MTBE \\
Reaction time & $3-30$ min \\
Oil-to-MTBE molar ratio & $1: 40$ \\
Temperature & $300-400^{\circ} \mathrm{C}$ \\
Pressure & 10,20 , and $30 \mathrm{MPa}$ \\
Reactor type & Spiral reactor \\
Reactor length & $10 \mathrm{~m}$ \\
\hline
\end{tabular}

the obtained products were collected. The experimental conditions are presented in Table 1.

The residence time was determined using Eq. (1), taking the reactor volume, the density of the canola oil and MTBE at the reaction temperature and pressure, and the mass flow rate of the canola oil and MTBE into consideration. It was assumed that there was no volume change caused by mixing.

(Residence time [min])

$$
=\frac{\left(\text { Reactor volume }\left[\mathrm{dm}^{3}\right]\right)}{\frac{(\text { Mass flow rate of oil } / \mathrm{min})}{\left(\text { Density of oil }\left[\mathrm{g} / \mathrm{dm}^{3}\right]\right)}+\frac{(\text { Mass flow rate of MTBE }[\mathrm{g} / \mathrm{min}])}{\text { (Density of MTBE } \left.\left[\mathrm{g} / \mathrm{dm}^{3}\right]\right)}}
$$

\subsection{Analysis}

Gas chromatography (GC-390B; GL Sciences) equipped with an MET-Biodiesel column with an integrated $2 \mathrm{~m}$ guard column (Sigma Aldrich, 28668-U) and a flameionization detector (FID) was employed to analyze the products. Argon was used as the carrier gas. The temperature program began at $50^{\circ} \mathrm{C}$ and the temperature was held for $1 \mathrm{~min}$. The temperature was raised at $15^{\circ} \mathrm{C} /$ $\min$ to $250^{\circ} \mathrm{C}$ and was kept at this temperature for $10 \mathrm{~min}$. Finally, the temperature was raised to $380^{\circ} \mathrm{C}$ at $15^{\circ} \mathrm{C} / \mathrm{min}$ and was held for $5 \mathrm{~min}$. The temperatures of the injector and detector were both set at $380^{\circ} \mathrm{C}$. Both standard and sample injection volumes were $1 \mu \mathrm{L}$, and peak identification was carried out via a comparison of retention times between the standard and sample compounds. Tricaprin was used as an internal standard.

Experimental FAME yields were calculated by dividing the moles of FAME product by the moles of fatty acid groups in the initial triglyceride (TG), as shown in Eq. (2). (Product yield)

$$
=\frac{(\mathrm{Mol} \text { of product FAME) }}{(\mathrm{Mol} \text { of fatty acid group in initial TG) }}
$$

\subsection{Reagents and materials}

All reagents were used without further treatment. The canola oil feedstock was a commercial one produced by J-Oil Mills (Tokyo, Japan). MTBE was purchased from Nacalai Tesque Inc. (Kyoto, Japan). Fatty acid methyl ester standards (methyl oleate, methyl linoleate, methyl linolenate, methyl palmitate, and methyl stearate) were purchased from Tokyo Chemical Industry Co. Ltd. (TCI, Tokyo). Triolein, diolein, and monoolein standards were purchased from Nacalai Tesque Inc., Sigma-Aldrich Co. (Japan), and TCI, respectively. The GTBE standard was purchased from Sigma-Aldrich, Co. The reagents used for GC standard preparation (tricaprin and n-hexane) were of analytical grade.

\section{Results and discussion}

\subsection{Effect of pressure on FAME yield}

To investigate the effect of pressure on FAME yield, the transesterification of canola oil and MTBE was carried out by varying the pressure from 10 to $30 \mathrm{MPa}$ at a fixed oil-to-MTBE molar ratio of 1:40 and a temperature range of $300-400^{\circ} \mathrm{C}$. The results for 10,20 , and $30 \mathrm{MPa}$ are shown in Figs. 2, 3, and 4, respectively.

At $10 \mathrm{MPa}$, a longer reaction time and a higher temperature resulted in a higher FAME yield. It is observed that at 300 and $350{ }^{\circ} \mathrm{C}$, FAME yields were still relatively low. FAME yields of 0.45 and $0.53 \mathrm{~mol} / \mathrm{mol}$ were obtained within $25 \mathrm{~min}$ at 300 and $350{ }^{\circ} \mathrm{C}$, respectively. The complete conversion to FAME was observed at $400{ }^{\circ} \mathrm{C}$ Under this condition, an FAME yield of $0.99 \mathrm{~mol} / \mathrm{mol}$ was obtained in the short reaction time of $5 \mathrm{~min}$.

At $20 \mathrm{MPa}$, a slight change in the conversion

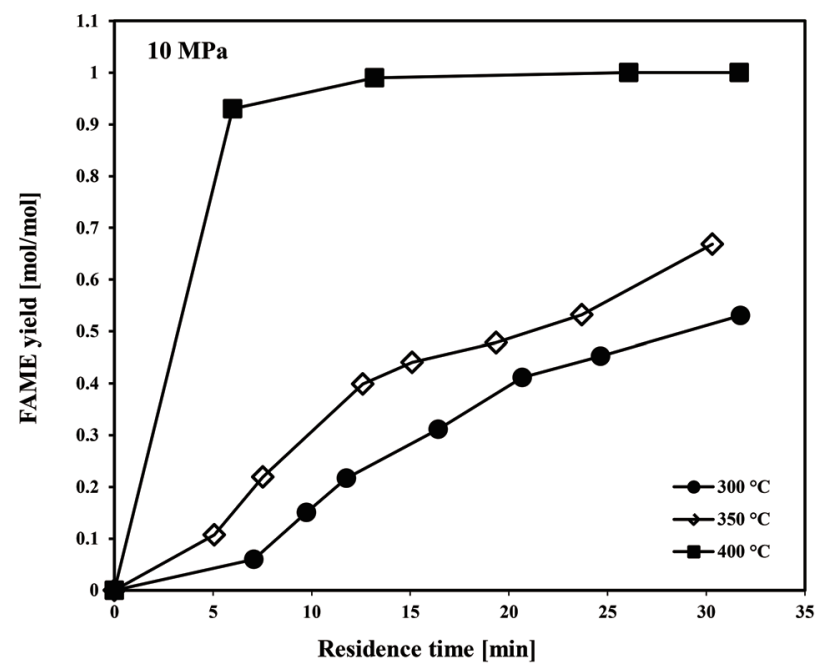

Fig. 2 FAME yield at $10 \mathrm{MPa}$ (Experimental conditions: $10 \mathrm{MPa}$, oil-to-MTBE molar ratio of 1:40) 


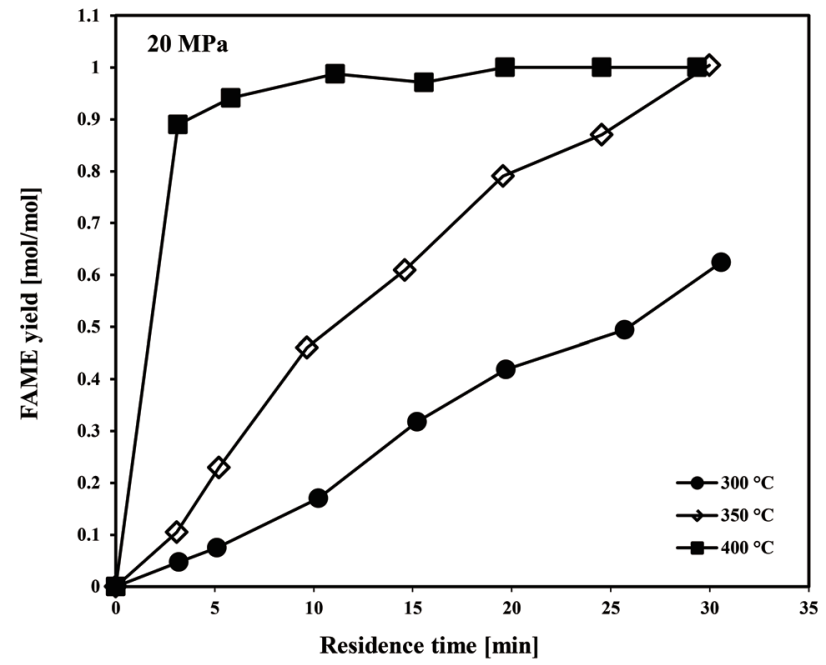

Fig. 3 FAME yield at $20 \mathrm{MPa}$ (Experimental conditions: $20 \mathrm{MPa}$, oil-to-MTBE molar ratio of 1:40)

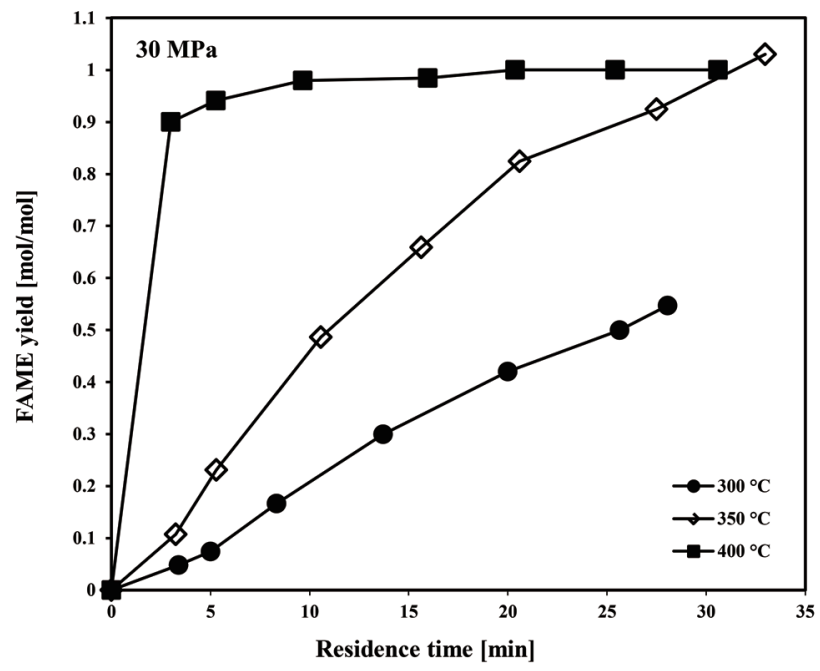

Fig. 4 FAME yield at $30 \mathrm{MPa}$ (Experimental conditions: $30 \mathrm{MPa}$, oil-to-MTBE molar ratio of 1:40)

of canola oil to FAME was observed at the milder temperature, i.e., $350{ }^{\circ} \mathrm{C}$. At this temperature, FAME yields of about 0.79 and $0.87 \mathrm{~mol} / \mathrm{mol}$ were obtained after transesterification times of 20 and $25 \mathrm{~min}$, respectively. Transesterification was almost complete within $30 \mathrm{~min}$, with almost all canola oil converted to FAME. At $400^{\circ} \mathrm{C}$, the complete conversion was observed in the initial time of 5 min and the yield was relatively constant thereafter.

At $30 \mathrm{MPa}$, the FAME yields obtained were almost the same as $20 \mathrm{MPa}$ at the same temperature and residence time. Again, at $400^{\circ} \mathrm{C}$, complete conversion was observed in $5 \mathrm{~min}$.

\subsection{Concentration change with time}

To quantitatively evaluate the effect of pressure, the concentration change over time should be discussed. Concentration changes of TG, GTBE, and FAME at 300, 350 , and $400^{\circ} \mathrm{C}$ are shown in Figs. 5, 6, and 7, respectively. As is expected, TG concentration decreases with residence time, while that of GTBE and FAME increases. At any temperature, the effect of pressure is not clearly
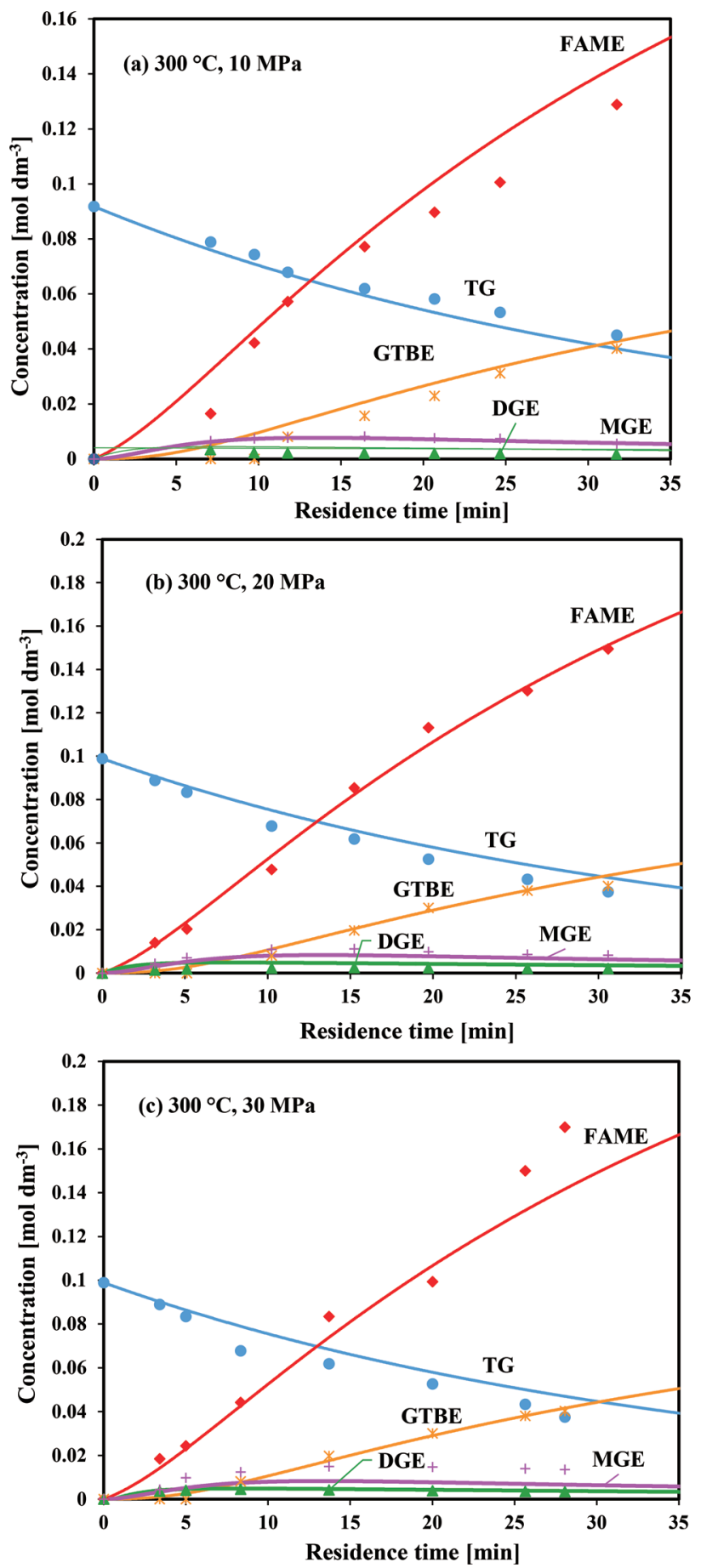

Fig. 5 Effect of pressure on TG consumption and GTBE yield at $300^{\circ} \mathrm{C}$ 

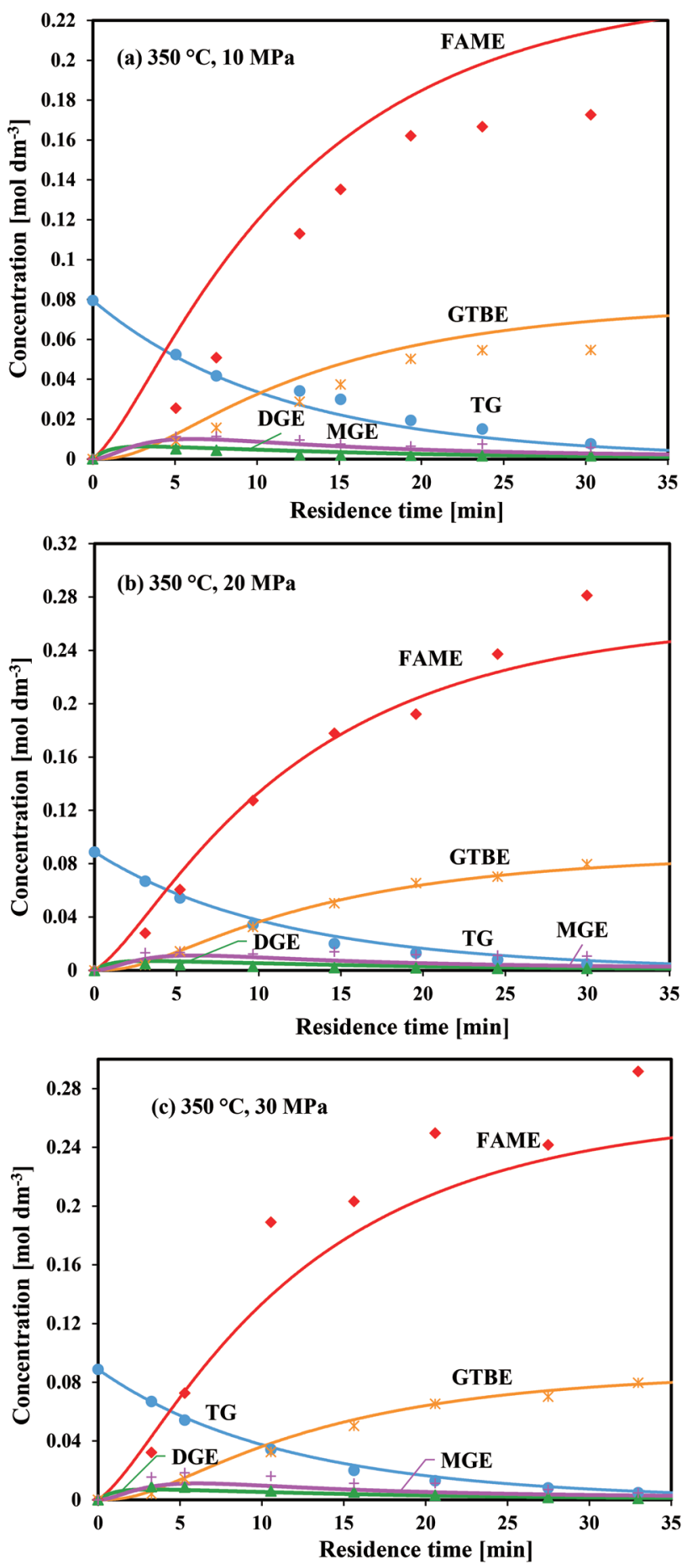

Fig. 6 Effect of pressure on TG consumption and GTBE yield at $350^{\circ} \mathrm{C}$

observed. This may appear surprising, considering that transesterification reaction proceeds between $\mathrm{TG}$ and MTBE, and thus the reaction order should be higher than unity. However, the density change of MTBE corresponding to the pressure change employed here is at most $6 \%$. This explains why the pressure effect was not observed in this study. It could be also noticed from Figs. 5-7 that the effect
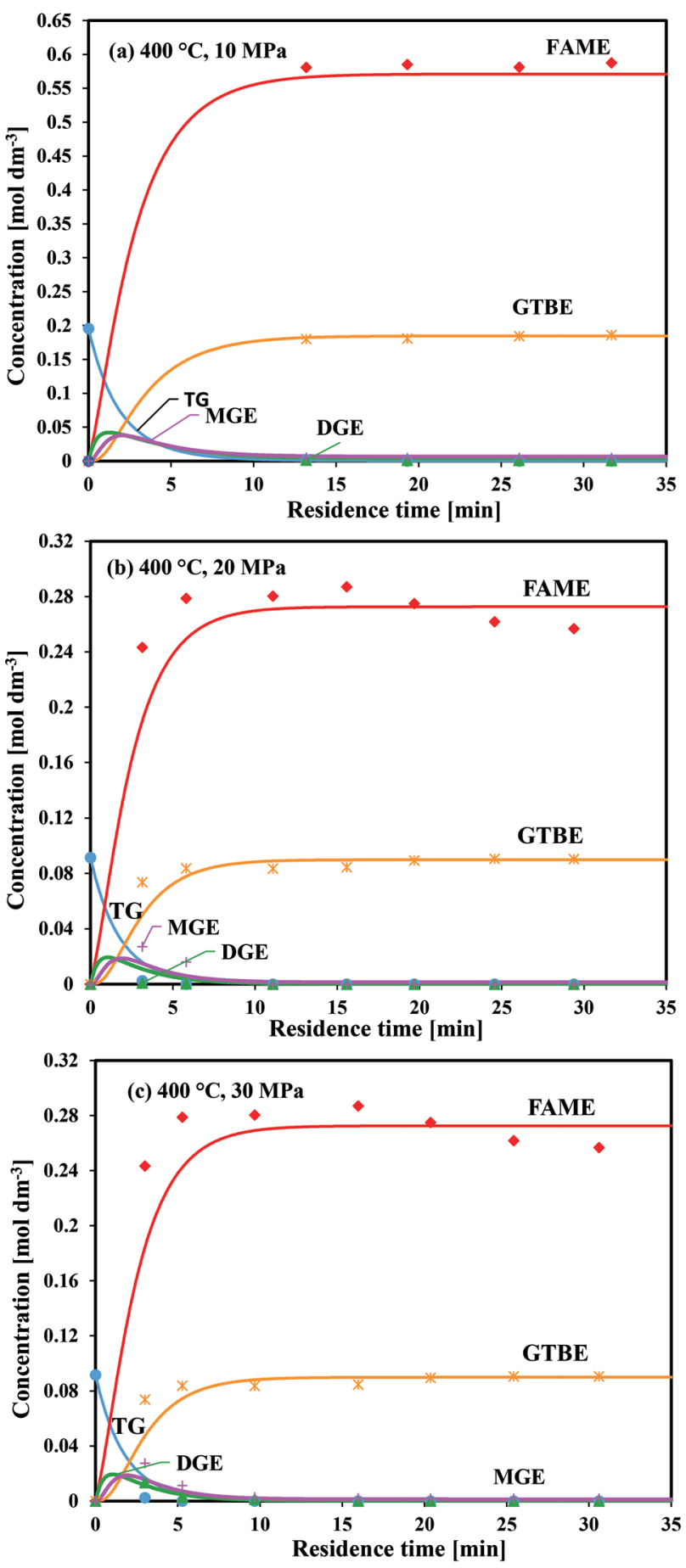

Fig. 7 Effect of pressure on TG consumption and GTBE yield at $400^{\circ} \mathrm{C}$

of pressure on intermediate compounds concentration was also negligible. At $300^{\circ} \mathrm{C}$, the concentrations of DGE and MGE were relatively constant with time. At 350 and $400^{\circ} \mathrm{C}$, the DGE and MGE concentrations increased up to $5 \mathrm{~min}$ but decreased afterward, indicating that these compounds were converted into FAME and GTBE. 


\subsection{Effect of pressure on reaction kinetics}

The kinetics of canola oil conversion to FAME has been reported in our previous paper ${ }^{4}$. However, the effect of pressure on reaction kinetics has not been reported yet. Briefly, the transesterification of canola oil with MTBE is assumed to occur in three consecutive reactions, as presented in Fig. 8. In the first step, TG reacts with MTBE to generate FAME and diglyceride mono tert-butyl ether (DGE). The intermediate DGE then reacts with MTBE to generate FAME and monoglyceride di tert-butyl ether (MGE). Lastly, the reaction of MGE and MTBE yields FAME and GTBE.

Having deduced the reaction mechanisms above, the next step is to fit the experimental data with a kinetic model with the aim of elucidating its kinetic parameters. The rate equation for each reaction shown above is assumed

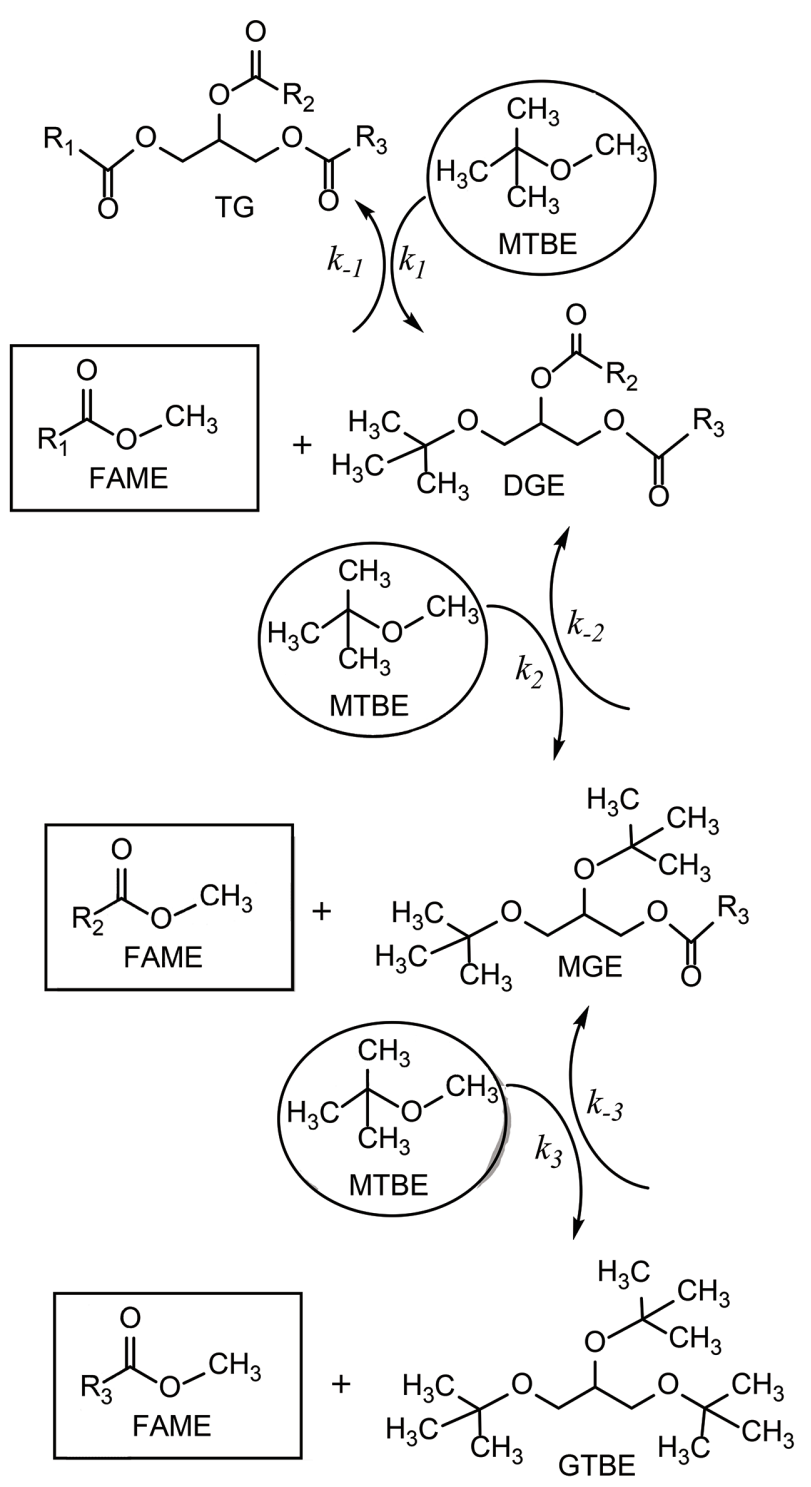

Fig. 8 Reaction mechanism of biodiesel production using MTBE to follow second-order kinetics, and the rate of change in concentration can thus be expressed by the differential rate equations shown in Eqs. (3) - (8).

$$
\begin{aligned}
\frac{d[\mathrm{TG}]}{d t}=- & k_{1}[\mathrm{TG}][\mathrm{MTBE}]+k_{-1}[\mathrm{FAME}][\mathrm{DGE}] \\
\frac{d[\mathrm{DGE}]}{d t}= & k_{1}[\mathrm{TG}][\mathrm{MTBE}]-k_{-1}[\mathrm{FAME}][\mathrm{DGE}] \\
& -k_{2}[\mathrm{DGE}][\mathrm{MTBE}]+k_{-2}[\mathrm{FAME}][\mathrm{MGE}] \\
\frac{d[\mathrm{MGE}]}{d t}= & k_{2}[\mathrm{DGE}][\mathrm{MTBE}]-k_{-2}[\mathrm{FAME}][\mathrm{MGE}] \\
& -k_{3}[\mathrm{MGE}][\mathrm{MTBE}]+k_{-3}[\mathrm{FAME}][\mathrm{GTBE}] \\
\frac{d[\mathrm{FAME}]}{d t}= & k_{1}[\mathrm{TG}][\mathrm{MTBE}]-k_{-1}[\mathrm{FAME}][\mathrm{DGE}] \\
& +k_{2}[\mathrm{DGE}][\mathrm{MTBE}]-k_{-2}[\mathrm{FAME}][\mathrm{MGE}] \\
& +k_{3}[\mathrm{MGE}][\mathrm{MTBE}]-k_{-3}[\mathrm{FAME}][\mathrm{GTBE}] \\
\frac{d[\mathrm{MTBE}]}{d t}= & -k_{1}[\mathrm{TG}][\mathrm{MTBE}]+k_{-1}[\mathrm{FAME}][\mathrm{DGE}] \\
& -k_{2}[\mathrm{DGE}][\mathrm{MTBE}]+k_{-2}[\mathrm{FAME}][\mathrm{MGE}] \\
& -k_{3}[\mathrm{MGE}][\mathrm{MTBE}]+k_{-3}[\mathrm{FAME}][\mathrm{GTBE}] \\
\frac{d[\mathrm{GTBE}]}{d t}= & k_{3}[\mathrm{MGE}][\mathrm{MTBE}]-k_{-3}[\mathrm{FAME}][\mathrm{GTBE}]
\end{aligned}
$$

where [TG] is the triglyceride concentration [mol $\left.\mathrm{dm}^{-3}\right],[\mathrm{DGE}]$ is the diglyceride mono tert-butyl ether concentration [mol dm${ }^{-3}$, [MGE] is the monoglyceride di tertbutyl ether concentration $\left[\mathrm{mol} \mathrm{dm}{ }^{-3}\right],[\mathrm{MTBE}]$ is the tertbutyl methyl ether concentration $\left[\mathrm{mol} \mathrm{dm}{ }^{-3}\right],[F A M E]$ is the fatty acid methyl ester concentration [mol dm${ }^{-3}$, [GTBE] is the tert-butyl glycerol ether concentration $\left[\mathrm{mol} \mathrm{dm}^{-3}\right], k_{\mathrm{i}}$ is the reaction rate constants $\left[\mathrm{dm}^{3} \mathrm{~mol}^{-1} \mathrm{~min}^{-1}\right]$, and $t$ is the residence time [min].

The least-squares error (LSE) method giving the best fitting between experimental and calculated values was used for the determination of the rate constants. Table 2 shows the kinetic parameters identified for the reaction pathways. All of the reaction rate constants increased with temperature, as expected. However, the reaction rate constants were not affected significantly by pressure.

Having determined the reaction rate constants at different temperature, the activation energy and preexponential factor were calculated via an Arrhenius equation. By assuming there is no pressure effect, the Arrhenius plot was derived by taking the average reaction rate constants of all pressures. The Arrhenius plots of the individual rate constants are shown in Fig. 9. Table 3 shows the activation energy $\left(E_{a}\right)$ and pre-exponential factor (A) for the detailed kinetic analysis. Activation energies between 31.83 and $100.19 \mathrm{~kJ} \mathrm{~mol}^{-1}$ were determined. Concentration changes of TG, GTBE, and FAME were calculated using these reaction parameters. The results are shown in Figs. $5-7$ by a smooth line. The agreement between calculation 
Table 2 Kinetic parameters obtained for the reaction pathways in the pressure range of 10-30 MPa

\begin{tabular}{|c|c|c|c|c|c|c|c|c|c|c|}
\hline \multirow{2}{*}{$\begin{array}{c}\text { Kinetic parameters } \\
{\left[\mathrm{dm}^{3} \mathrm{~mol}^{-1} \mathrm{~min}^{-1}\right]}\end{array}$} & \multirow{2}{*}{ Denotation } & \multicolumn{3}{|c|}{$10 \mathrm{MPa}$} & \multicolumn{3}{|c|}{$20 \mathrm{MPa}$} & \multicolumn{3}{|c|}{$30 \mathrm{MPa}$} \\
\hline & & $300^{\circ} \mathrm{C}$ & $350^{\circ} \mathrm{C}$ & $400^{\circ} \mathrm{C}$ & $300^{\circ} \mathrm{C}$ & $350^{\circ} \mathrm{C}$ & $400^{\circ} \mathrm{C}$ & $300^{\circ} \mathrm{C}$ & $350^{\circ} \mathrm{C}$ & $400^{\circ} \mathrm{C}$ \\
\hline$k_{1}$ & $\mathrm{TG} \rightarrow \mathrm{DGE}$ & 0.0060 & 0.0202 & 0.1655 & 0.0076 & 0.0256 & 0.1650 & 0.0078 & 0.0268 & 0.1660 \\
\hline$k_{-1}$ & $\mathrm{DGE} \rightarrow \mathrm{TG}$ & 0.0249 & 0.0395 & 0.0580 & 0.0211 & 0.0403 & 0.0600 & 0.0201 & 0.0400 & 0.0590 \\
\hline$k_{2}$ & $\mathrm{DGE} \rightarrow \mathrm{MGE}$ & 0.1374 & 0.2405 & 0.4555 & 0.1174 & 0.2519 & 0.4555 & 0.1207 & 0.2359 & 0.4575 \\
\hline$k_{-2}$ & $\mathrm{MGE} \rightarrow \mathrm{DGE}$ & 0.4700 & 0.8400 & 1.3400 & 0.4500 & 0.8400 & 1.3400 & 0.4450 & 0.8300 & 1.3200 \\
\hline$k_{3}$ & $\mathrm{MGE} \rightarrow \mathrm{GTBE}$ & 0.0602 & 0.1047 & 0.3883 & 0.0625 & 0.1262 & 0.3436 & 0.0630 & 0.1346 & 0.3408 \\
\hline$k_{-3}$ & $\mathrm{GTBE} \rightarrow \mathrm{MGE}$ & 0.0151 & 0.0302 & 0.0550 & 0.0154 & 0.0305 & 0.0600 & 0.0164 & 0.0296 & 0.0580 \\
\hline
\end{tabular}
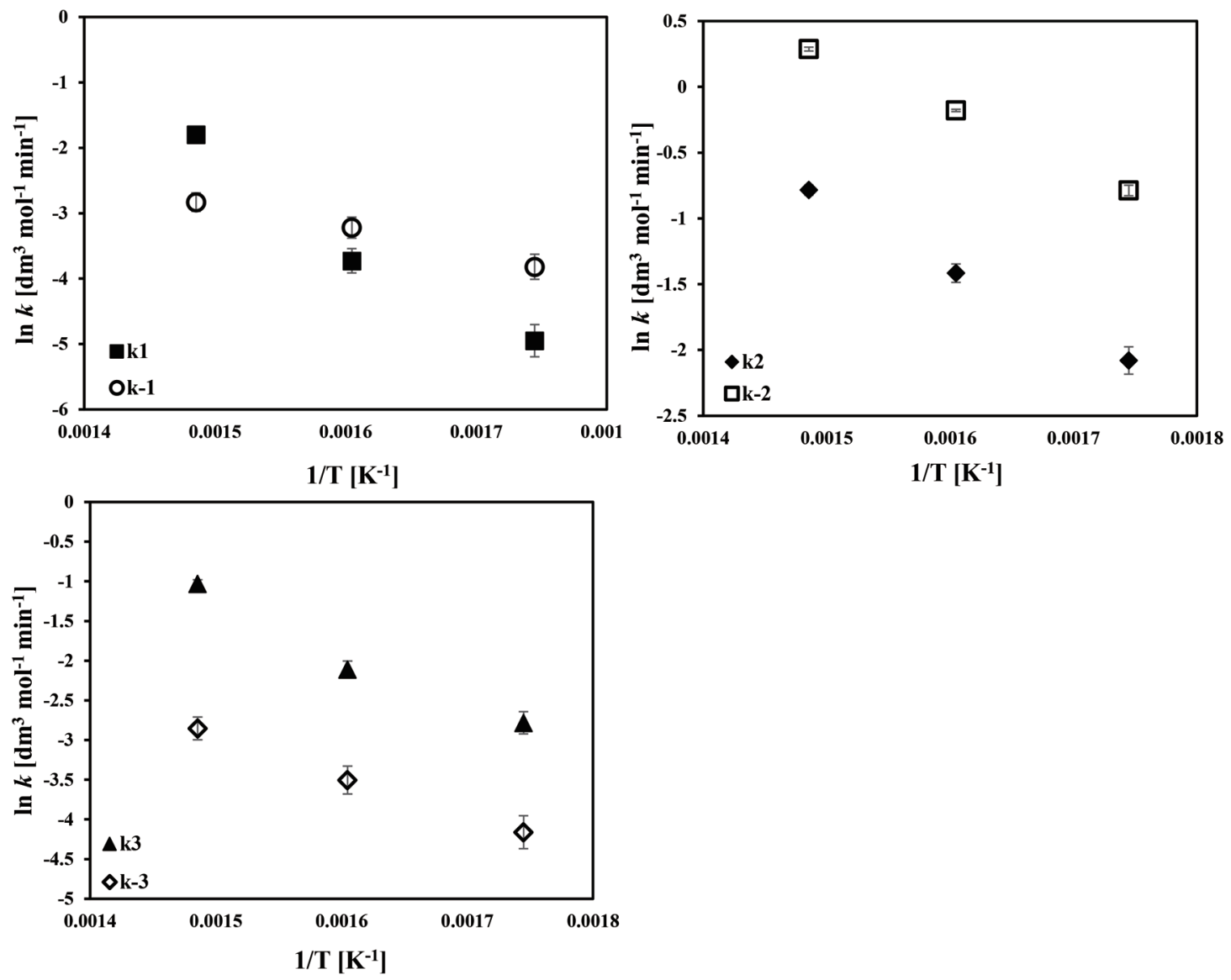

Fig. 9 Arrhenius plots of the individual reaction rate constants

Table 3 Activation energy and pre-exponential factor

\begin{tabular}{llll}
\hline \multicolumn{1}{c}{ Reaction direction } & $\begin{array}{c}\text { Reaction rate constant } \\
{\left[\mathrm{dm}^{3} \mathrm{~mol}^{-1} \mathrm{~min}^{-1}\right]}\end{array}$ & $\begin{array}{c}\text { Activation energy, } E a \\
{\left[\mathrm{~kJ} \mathrm{~mol}{ }^{-1}\right]}\end{array}$ & $\begin{array}{c}\text { Pre-exponential factor, } A \\
{\left[\mathrm{dm}^{3} \mathrm{~mol}^{-1} \mathrm{~min}^{-1}\right]}\end{array}$ \\
\hline $\mathrm{TG} \rightarrow \mathrm{DGE}$ & $k_{1}$ & 100.19 & $8.29 \times 10^{6}$ \\
$\mathrm{DGE} \rightarrow \mathrm{TG}$ & $k_{-1}$ & 31.83 & 17.83 \\
$\mathrm{DGE} \rightarrow \mathrm{MGE}$ & $k_{2}$ & 41.49 & 746.95 \\
$\mathrm{MGE} \rightarrow \mathrm{DGE}$ & $k_{-2}$ & 34.54 & 645.42 \\
$\mathrm{MGE} \rightarrow \mathrm{GTBE}$ & $k_{3}$ & 55.73 & $6.83 \times 10^{3}$ \\
$\mathrm{GTBE} \rightarrow \mathrm{MGE}$ & $k_{-3}$ & 41.87 & 100.57 \\
\hline
\end{tabular}

and experimental results is good that was proven by the high value of coefficient of determination $\left(r^{2}=0.9864\right)$ as shown in Fig. 10. In addition, the effectiveness of the reaction rate parameters shown in Table 3 is clear.

Two more points should be mentioned regarding the effect of pressure. First, the negligible effect observed in this study does not agree with some of the previous studies in which the effect of pressure was large at lower pressure, and the pressure increase no longer affected the yield at higher pressures ${ }^{7)} \sim 10$ ). Therefore, the pressure 


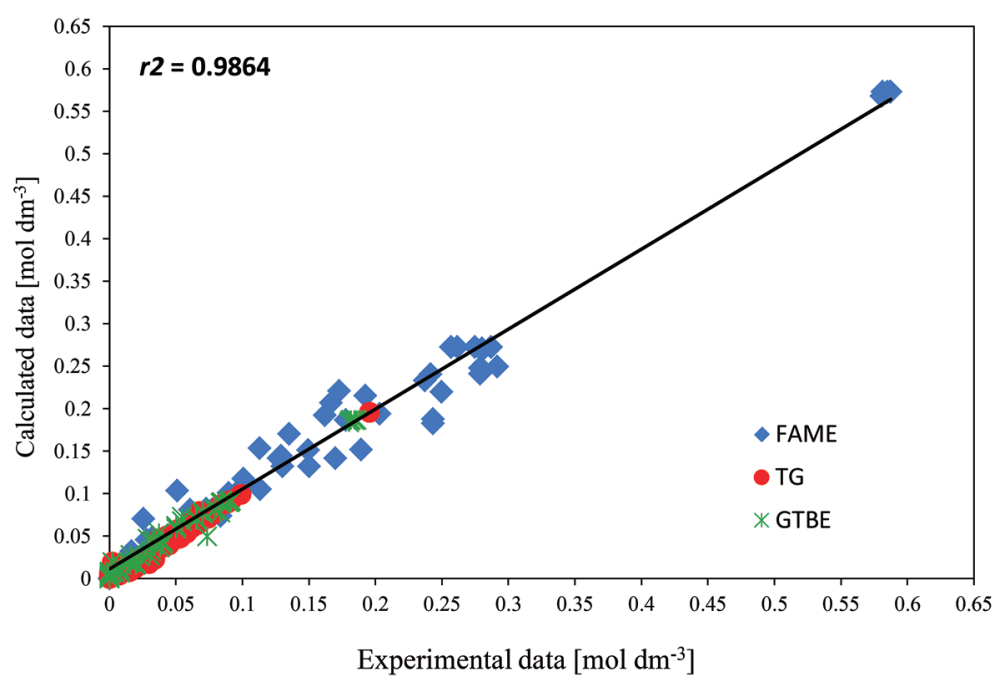

Fig. 10 Comparison of experimental and calculated data for concentrations of FAME, TG, and GTBE

range employed in this study may have been sufficiently high, and may have corresponded to the state in which a pressure increase does not affect the yield (as in previous studies). The fact that the critical pressure of MTBE (3.45 MPa) is much lower than those of methanol (8.09 $\mathrm{MPa})$ and ethanol (6.14 $\mathrm{MPa})$ supports this idea. In terms of reduced pressure, the pressure range employed in this study is almost twice as large as those employed in the previous studies. Experiment at lower pressure should be interesting, and it will be our next target. What we want to point out here is that for the pressure range usually employed in the supercritical biodiesel production, no effect is observed for MTBE, which is different from the alcohols. Effect of pressure is sometimes discussed in relation with the solubility of oil to the solvent. However, it is for the subcritical region that phase separation is discussed, and in supercritical reactions discussed here, single phase reaction should be safely assumed.

Second, complete conversion of TG into FAME at sufficiently long residence time indicates that reaction equilibrium was on the FAME production side for the reaction system employed here. The oil-to-MTBE molar ratio of 1:40 employed in this study explains this. When a complete reaction takes place, the reaction between TG and MTBE to produce GTBE and FAME consumes 4 molecules to produce 4 molecules. Thus, this reaction equilibrium should not be affected by pressure. Therefore, it is reasonable to conclude that pressure has no effect on the final reaction product composition observed here.

\section{Conclusions}

The effect of pressure on biodiesel production using supercritical MTBE using a continuous reactor was examined. The pressure was changed in the range of 10-30 $\mathrm{MPa}$ at a temperature range of $300-400{ }^{\circ} \mathrm{C}$. The effect of pressure on reaction rate as well as final product composition was negligible for the conditions employed here. This negligible effect could be explained by the almost constant density of MTBE. The pre-exponential factors and activation energy for each reaction step as well as its reverse reaction have been determined.

\section{Acknowledgment}

OF gratefully acknowledges the financial support of Japan-Indonesia Presidential Scholarship (JIPS), the World Bank Institute.

\section{References}

1) Knothe, G.; Sharp, C. A.; Ryan, T.W. III., Energy Fuels, 20, 403-408 (2006)

2) Guo, Y.; Wei, H.; Yang, F.; Li, D.; Fang, W.; Lin, R., J. Hazard. Mater., 167, 625-629 (2009)

3) Kusdiana, D., Saka, S., Bioresour. Technol., 91, 289-295 (2004)

4) Farobie, O.; Yanagida, T.; Matsumura, Y., Fuel, 135, 172181 (2014).

5) Saka, S., Kusdiana, D., Fuel, 80, 225-231 (2001)

6) Antal, M. J., Jr.; Helsen, L. M.; Kouzu, M.; Lédé, J.; Matsumura, Y., J. Jpn. Inst. Energy, 93, 684-702 (2014)

7) Klepáčová, K.; Mravec, D.; Kaszonyi, A.; Bajus, M., Appl. Catal., A, 328, 1-13 (2007)

8) Frusteri, F.; Arena, F.; Bonura, G.; Cannilla, C.; Spadaro, L.; Di Blasi, O., Appl. Catal. A, 367, 77-83 (2009)

9) He, H.; Sun, S.; Wang, T.; Zhu, S., J. Am. Oil. Chem. Soc., 84, 399-404 (2007)

10) Warabi, Y.; Kusdiana, D.; Saka, S., Appl. Biochem. Biotech., 115, 793-801 (2004)

11) Ilham, Z., Saka, S., Fuel, 97, 670-677 (2012)

12) Goembira, F.; Saka, S., Bioresour. Technol., 131, 47-52 (2013) 